\title{
Optimal Clock Synchronization
}

\author{
T. K. SRIKANTH AND SAM TOUEG \\ Cornell University, Ithaca, New York
}

\begin{abstract}
We present a simple, efficient, and unified solution to the problems of synchronizing, initializing, and integrating clocks for systems with different types of failures: crash, omission, and arbitrary failures with and without message authentication. This is the first known solution that achieves optimal accuracy-the accuracy of synchronized clocks (with respect to real time) is as good as that specified for the underlying hardware clocks. The solution is also optimal with respect to the number of faulty processes that can be tolerated to achieve this accuracy.
\end{abstract}

Categories and Subject Descriptors: D.4.5 [Operating Systems]: Reliability-fault-tolerance; D.4.7 [Operating Systems]: Organization and Design-real-time systems

General Terms: Algorithms, Reliability, Theory

Additional Key Words and Phrases: Authentication, Byzantine failures, clock synchronization

\section{Introduction}

An important problem in distributed computing is that of synchronizing clocks in spite of faults. Given "hardware" clocks whose rate of drift from real time is within known bounds, synchronization consists of maintaining logical clocks that are never too far apart. Processes maintain these logical clocks by computing periodic adjustments to their hardware clocks.

Although the underlying hardware clocks have a bounded rate of drift from real time, the drift of logical clocks can exceed this bound. In other words, while synchronization ensures that logical clocks are close together, the accuracy of these logical clocks (with respect to real time) can be lower than that specified for hardware clocks. This reduction in accuracy might appear to be an inherent consequence of synchronization in the presence of failures. The rate of drift of faulty hardware clocks can be beyond the specified bounds, and correct logical clocks can be forced to drift with them. Furthermore, variation in message delivery times introduces uncertainty in evaluating values of clocks of other processes. All previous synchronization algorithms exhibit this reduction in clock accuracy $[3,4,7,9-12]$.

In this paper we show that accuracy need not be sacrificed in order to achieve synchronization. We present the first synchronization algorithm where logical clocks have the same accuracy as the underlying physical clocks. We show that no

This is an expanded version of a paper presented at the Fourth Annual ACM Symposium on Principles of Distributed Computing, Minaki, Canada, Aug. 1985. This work was supported in part by the National Science Foundation under grant MCS 83-03135.

Authors' address: Department of Computer Science, Cornell University, Ithaca, NY 14853.

Permission to copy without fee all or part of this material is granted provided that the copies are not made or distributed for direct commercial advantage, the ACM copyright notice and the title of the publication and its date appear, and notice is given that copying is by permission of the Association for Computing Machinery. To copy otherwise, or to republish, requires a fee and/or specific permission. (c) $1987 \mathrm{ACM} 0004-5411 / 87 / 0700-0626 \$ 01.50$ 
synchronization algorithm can achieve a better accuracy, and therefore our algorithm is optimal in this respect.

In previous results a different algorithm has been derived for each model of failure. In contrast, ours is a unified solution to clock synchronization for several models of failure: crash, omission, or arbitrary (i.e., "Byzantine") failures with and without message authentication. With simple modifications the solution also provides for initial clock synchronization and the integration of new clocks.

To overcome the overwhelming difficulty of developing an optimal synchronization algorithm in a system with arbitrary failures, we first assume that the system provides message authentication $[2,7]$. With this assumption we are able to derive an algorithm that is both simple and efficient. We then replace signed communication with a broadcast primitive that achieves those properties of message authentication required by the algorithm [14]. This automatically results in an equivalent algorithm for systems with arbitrary failures without digital signatures. This solution is then simplified for crash and omission failures.

We show that to achieve optimal accuracy, fewer than half the clocks in the system can be faulty. With arbitrary failures and in the absence of authentication, synchronization can be achieved only if fewer than a third of the clocks in the system are faulty [3]. For all the models of failure that we consider, our algorithms are optimal with respect to the number of faulty clocks they can tolerate. ${ }^{1}$

The solution presented in this paper is simple and efficient, and can be easily implemented [1]. Its message complexity is similar to those previously published. Further comparisons with previous results are presented in Section 8.

The paper is organized as follows: We describe the system model in Section 2. In Section 3 we describe an authenticated synchronization algorithm that achieves optimal accuracy, and we derive bounds on the number of faults that can be tolerated to achieve this accuracy. In Section 4 we present a broadcast primitive that achieves properties of authenticated broadcasts and use it to get a nonauthenticated synchronization algorithm. Initialization and integration are described in Section 5. Crash and omission models of failure are considered in Section 6. In Section 7 we describe how processes can maintain a single continuous clock. Discussion and concluding remarks are presented in Sections 8 and 9.

\section{The Model}

We consider a system of distributed processes that communicate through a reliable, error-free, and fully connected point-to-point message system (relaxing the connectivity requirement is discussed in Section 8). Each process has a physical "hardware" clock and computes its logical time by adding a locally determined adjustment to this physical clock.

The notation used here closely follows that in [7]. Variables and constants associated with real time are in lowercase, and those corresponding to the logical time of a process are in uppercase. The following assumptions are made about the system:

A1. The rate of drift of physical clocks from real time is bounded by a known constant $\rho>0$. That is, if $R_{i}(t)$ is the reading of the physical clock of process $i$ at time $t$, then for all $t_{2} \geq t_{1}$,

$$
(1+\rho)^{-1}\left(t_{2}-t_{1}\right) \leq R_{i}\left(t_{2}\right)-R_{i}\left(t_{1}\right) \leq(1+\rho)\left(t_{2}-t_{1}\right) .
$$

${ }^{1}$ The authenticated algorithm in [7] can overcome an arbitrary number of failures. However, the accuracy of the synchronized clocks is not optimal. 
Thus, correct physical clocks are within a linear envelope of real time. We also see that the rate of drift between clocks is bounded by $d r=$ $\rho(2+\rho) /(1+\rho)$.

A2. There is a known upper bound $t_{\text {del }}$ on the time required for a message to be prepared by a process, sent to all processes and processed by the recipients of the message.

A process is faulty if it deviates from its algorithm or if its physical clock violates assumption A1; otherwise it is said to be correct. Faulty processes may also collude to prevent correct processes from achieving synchronization. We use the term correct clock to refer to the logical clock of a correct process.

Resynchronization proceeds in rounds, a period of time in which processes exchange messages and reset their clocks. To simplify the presentation and analysis, we adopt the standard convention that a process $i$ starts a new logical clock, denoted $C_{i}^{k}$, after the $k$ th resynchronization. In practice, this introduces some ambiguity as to which clock a process should use when an external application requests the time. In Section 7 we remove this ambiguity by showing how each process can maintain a single continuous logical clock. Define $b e g^{k}$ and $e n d^{k}$ to be the real time at which the first and last correct process, respectively, start their $k$ th clocks. The period $\left[\right.$ beg $^{k}$, end $\left.^{k}\right]$ is the $k$ th resynchronization period.

Given the above assumptions, a synchronization algorithm is one that satisfies the following conditions for all correct clocks $i$ and $j$, all $k \geq 1$, and $t \in$ $\left[\right.$ end ${ }^{k}$, end $\left.d^{k+1}\right]$ :

(1) Agreement. There exists a constant $D_{\max }$ such that

$$
\left|C_{i}^{k}(t)-C_{j}^{k}(t)\right| \leq D_{\max }
$$

(2) Accuracy. There exists a constant $\gamma$ such that, for any execution of the algorithm,

$$
(1+\gamma)^{-1} t+a \leq C_{i}^{k}(t) \leq(1+\gamma) t+b
$$

for some constants $a$ and $b$ that depend on the initial conditions of this execution.

The agreement condition asserts that the maximum deviation between correct logical clocks is bounded. The accuracy condition states that correct logical clocks are within a linear envelope of real time.

Note that $\gamma$ is a bound on the rate of drift of logical clocks from real time and hence is a measure of their accuracy with respect to real time. We are interested in synchronization algorithms that minimize $\gamma$. In Theorem 2 we show that $\gamma$ cannot be smaller than $\rho$, the bound on the accuracy of physical clocks. Therefore we are interested in algorithms satisfying the following conditions:

(3) Optimal accuracy. For any execution of the algorithm, for all correct clocks $i$, all $k \geq 1$, and $t \in\left[\right.$ end $^{k}$, end $\left.d^{k+1}\right]$,

$$
(1+\rho)^{-1} t+a \leq C_{i}^{k}(t) \leq(1+\rho) t+b
$$

for some constants $a$ and $b$ that depend on the initial conditions of this execution. 


\section{The Authenticated Algorithm}

The following is an informal description of a synchronization algorithm for systems with $n$ processes of which at most $f$ are faulty. The algorithm requires that $n \geq$ $2 f+1$ and that messages are authenticated. Informally, authentication prevents a faulty process from changing a message it relays, or introducing a new message into the system and claiming to have received it from some other process.

Let $P$ be the logical time between resynchronizations. A process expects the $k$ th resynchronization, for $k \geq 1$, at time $k P$ on its logical clock. When $C^{k-1}(t)=k P$ it broadcasts a signed message of the form (round $k$ ), indicating that it is ready to resynchronize. When a process receives such a message from $f+1$ distinct processes, it knows that at least one correct process is ready to resynchronize. It is then said to accept the message, and decides to resynchronize, even if its logical clock has not yet reached $k P$. A process resynchronizes by starting its $k$ th clock, setting it to $k P+\alpha$, where $\alpha$ is a constant. To ensure that clocks are never set back, $\alpha$ is chosen to be greater than the increase in $C^{k-1}$ since the process sent a (round $k$ ) message. After resynchronizing, the process also relays the $f+1$ signed (round $k$ ) messages to all other processes to ensure that they also resynchronize. The algorithm is described in Figure 1. We show that it achieves agreement and accuracy. We later modify it to achieve optimal accuracy.

3.1 Proof of Correctness: Agreement. We first show that the algorithm achieves the agreement property. Define ready ${ }^{k}$ to be the earliest (real) time at which any correct process sends a (round $k$ ) message. We assume that the clocks $C^{0}$ of correct processes are synchronized; that is, at ready ${ }^{1}$ all correct processes are using clock $C^{0}$ and, for all correct processes $i$ and $j, \mid C_{i}^{0}\left(\right.$ ready $\left.^{1}\right)-C_{j}^{0}\left(\right.$ ready $\left.^{1}\right) \mid \leq$ $D_{\max }$. In Section 5 we describe an algorithm for achieving this initial synchronization. For ease of presentation, we assume that the maximum permitted deviation between correct logical clocks, $D_{\max }$, is a given constraint.

LEMMA 1. The kth resynchronization period is bounded in size. That is, there exists a constant $d_{\min }$ such that, for $k \geq 1$, end $d^{k}-$ beg $^{k} \leq d_{\text {min }}$.

PROOF. Let $p$ be the first correct process to start its $k$ th clock. By definition, this occurs at $b{ }^{k}$. Process $p$ must have received $f+1$ signed (round $k$ ) messages. Since it relays all these messages, every correct process receives them and accepts the message (round $k$ ) by time $b^{k} g^{k}+t_{\text {del }}$. Hence every correct process starts its $k$ th clock by time $b e g^{k}+t_{\text {del }}$. By setting $d_{\min }=t_{\text {del, }}$, we get $e n d^{k} \leq b e g^{k}+d_{\min }$.

LEMMA 2. At the end of the kth resynchronization period, correct clocks differ by at most $d_{\min }(1+\rho)$. That is, for $k \geq 1$ and for all correct processes $i$ and $j$, $\mid C_{i}^{k}\left(\right.$ end $\left.^{k}\right)-C_{j}^{k}\left(\right.$ end $\left.^{k}\right) \mid \leq d_{\min }(1+\rho)$.

ProOF. By Lemma 1, end $d^{k}-b e g^{k} \leq d_{\min }$. Therefore the last correct process to start its $k$ th clock does so within $d_{\min }$ of the first correct clock doing so, and in this period, the first clock could have drifted by at most $\rho d_{\text {min. }}$. Thus, at $e n d^{k}$, the difference between correct clocks is at most $d_{\min }(1+\rho)$.

Lemma 3. No correct process starts its $k$ th clock until at least one correct process is ready to do so, that is, beg ${ }^{k} \geq$ ready $^{k}$ for $k \geq 1$.

PROOF. The first correct process to start its $k$ th clock does so only when it accepts a (round $k$ ) message, that is, only when it receives (round $k$ ) messages from at least $f+1$ processes. Since at least one correct process must have sent a (round $k$ ) message, beg ${ }^{k} \geq$ ready $^{k}$. 


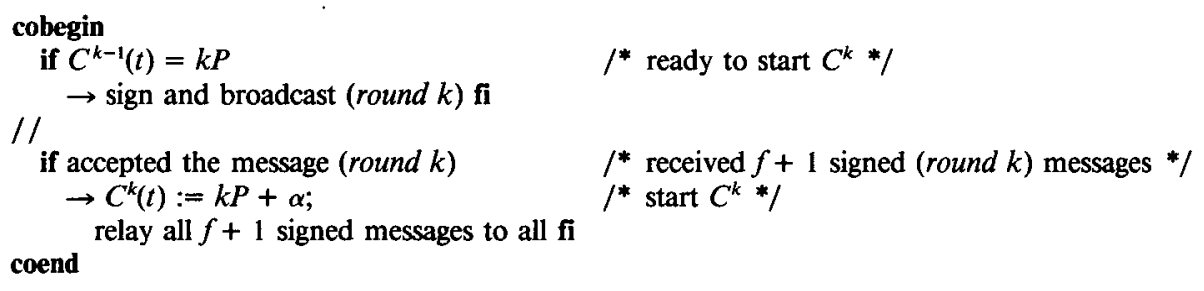

FIG. 1. An authenticated algorithm for clock synchronization for process $p$ for round $k$.

Assume that the following conditions hold for some $k \geq 1$.

S1. By ready ${ }^{k}$, all correct processes have already started clock $C^{k-1}$.

S2. For correct processes $i$ and $j, \mid C_{i}^{k-1}\left(\right.$ ready $\left.^{k}\right)-C_{j}^{k-1}\left(\right.$ ready $\left.^{k}\right) \mid \leq D_{\max }$.

With these assumptions we prove the following lemmas:

Lemma 4. All correct processes start their kth clocks soon after one correct process is ready to do so. Specifically, end ${ }^{k}-$ ready ${ }^{k} \leq(1+\rho) D_{\max }+t_{d e l}$.

Proof. The first correct process to send a (round $k$ ) message does so at ready ${ }^{k}$. By S2, the slowest correct clock is no more than $D_{\max }$ behind. Hence every correct process sends a (round $k$ ) message no later than $(1+\rho) D_{\max }$ after ready, and therefore every correct process starts its $k$ th clock within a further $t_{\text {del. }}$. Thus end ${ }^{k}$ $-\operatorname{read} y^{k} \leq(1+\rho) D_{\max }+t_{\mathrm{del}}$.

By Lemma 4, the real time that elapses from the time a correct process sends a (round $k$ ) message (when $C^{k-1}$ reads $k P$ ) to the time it starts $C^{k}$ (setting it to $k P+\alpha)$ is at most $(1+\rho) D_{\max }+t_{\mathrm{dec}}$. Therefore, if $\alpha \geq\left[(1+\rho) D_{\max }+t_{\mathrm{del}}\right](1+\rho)$, then no correct process sets its logical clock backward. Henceforth we assume that $\alpha$ satisfies this relation.

Lemma 5. The period between resynchronizations is bounded. Specifically, $e^{\text {end }}{ }^{k+1}-$ end $^{k} \leq(P-\alpha)(1+\rho)+t_{\text {del }}$.

Proof. Every correct process that sends a (round $k+1)$ message does so no later than the time $(k+1) P$ on its clock, that is, no later than $(P-\alpha)(1+\rho)$ after $e n d^{k}$. Every process starts its $k+1$ st clock within a further $t_{\mathrm{del}}$, thus proving the lemma.

We now assume that $P$ satisfies the following two relations: $D_{\max } \geq[P(1+\rho)+$ $\left.t_{\mathrm{del}}\right] d r+d_{\min }(1+\rho)$ and $P>d_{\min }(1+\rho)+\alpha$. With these assumptions we prove the following lemmas:

Lemma 6. The maximum deviation between the kth logical clocks of correct processes $i$ and $j$ is bounded. That is, for $t \in\left[\right.$ end $^{k}$, end $\left.{ }^{k+1}\right],\left|C_{i}^{k}(t)-C_{j}^{k}(t)\right| \leq$ $D_{\max }$.

Proof. By Lemma 2, correct logical clocks are at most $d_{\min }(1+\rho)$ apart at end $^{k}$. By Lemma 5 , end $d^{k+1}-e d^{k} \leq(P-\alpha)(1+\rho)+t_{\mathrm{del}}$, and clocks of correct processes can drift apart at a rate $d r$ in this interval. Thus, in the interval $\left[\right.$ end $^{k}$, end $\left.^{k+1}\right]$,

$$
\begin{aligned}
\left|C_{i}^{k}(t)-C_{j}^{k}(t)\right| & \leq\left[(P-\alpha)(1+\rho)+t_{\mathrm{det}}\right] d r+d_{\min }(1+\rho) \\
& \leq\left[P(1+\rho)+t_{\mathrm{del}}\right] d r+d_{\min }(1+\rho) \leq D_{\max }
\end{aligned}
$$

since $P$ satisfies the relation $D_{\max } \geq\left[P(1+\rho)+t_{\mathrm{del}}\right] d r+d_{\min }(1+\rho)$. 
LEMma 7. Synchronization periods do not overlap. That is, end ${ }^{k}<$ ready $y^{k+1} \leq$ beg $^{k+1}$.

ProOF. The first correct process to send a (round $k+1$ ) message does so no earlier than at real time $b e g^{k}+(P-\alpha) /(1+\rho)$. Therefore ready ${ }^{k+1} \geq$ beg $^{k}+$ $(P-\alpha) /(1+\rho)$. Hence, by Lemma 1 , read $y^{k+1} \geq e n d^{k}-d_{\min }+(P-\alpha) /(1+\rho)$. By Lemma 3, ready ${ }^{k+1} \leq b e g^{k+1}$. Thus end $d^{k}<$ ready $y^{k+1} \leq b e g^{k+1}$, since $P$ satisfies $P>d_{\min }(1+\rho)+\alpha$.

From the proofs of Lemmas 6 and 7, we see that $D_{\max }$ cannot be made arbitrarily small. The proof of Lemma 6 shows that $D_{\max } \geq\left[(P-\alpha)(1+\rho)+t_{\mathrm{del}}\right] d r+$ $d_{\min }(1+\rho)$. From Lemma 7 we see that $P-\alpha \geq d_{\min }(1+\rho)$. Therefore the smallest possible $D_{\max }$ that this algorithm can achieve is given by $D_{\max } \geq$ $d_{\min }(1+\rho)^{3}+t_{\mathrm{del}} d r$. Dolev et al. have shown that the real time between when clocks read the same value cannot be guaranteed to be better than $t_{\mathrm{del}} / 2$ [3]. Hence, when optimal accuracy is required, $D_{\max }$ must be at least $(1+\rho)^{-1} t_{\mathrm{del}} / 2$.

LEMMA 8. The algorithm in Figure 1 achieves agreement.

ProOF. If assumptions S1 and S2 hold for some $k \geq 1$, then Lemma 6 states that the agreement condition is satisfied for $k$. We now show, by induction on $k$, that $\mathbf{S} 1$ and $\mathbf{S} 2$ hold for all $k \geq 1$, and therefore agreement is satisfied for all $k \geq 1$. As stated earlier, our initialization algorithm will guarantee that S1 and S2 are true for the base case, $k=1$.

Assume that S1 and S2 are true for some $k$. By Lemma 7, end ${ }^{k}<r e a d y^{k+1} \leq$ $b e g^{k+1}$. Thus, at ready $y^{k+1}$, all correct processes use their $k$ th clocks. From Lemma 6 it follows that, at $t=r e a d y^{k+1}$ and for correct processes $i$ and $j$, $\left|C_{l}^{k}(t)-C_{j}^{k}(t)\right| \leq D_{\max }$. Thus $\mathbf{S} 1$ and $\mathbf{S} 2$ are true for $k+1$.

3.2 ProOF OF CORRECTNESS: ACCURACY. We now show that the algorithm achieves accuracy.

LEMMA 9. For any execution of the algorithm of Figure 1, there exists a constant $b$, such that, for all correct processes $i$, all $k \geq 1$, and for $t \in\left[\right.$ end $^{k}$, end $\left.d^{k+1}\right]$,

$$
C_{i}^{k}(t) \leq \frac{P}{P-\alpha}(1+\rho) t+b .
$$

ProOF. Let $E\left(t_{0}\right)$ be the set of executions of the algorithm in which ready ${ }^{1}=$ $t_{0}$. Consider an execution $e \in E\left(t_{0}\right)$ in which for all $k \geq 1$, ready ${ }^{k}=$ beg $^{k}$, and the clock of correct process $j, C_{j}^{k}$, is started by $b^{2} g^{k}$. In execution $e$ the physical clock of process $j$ runs at the maximum possible rate, that is, $(1+\rho)$ with respect to real time. It is clear that execution $e$ is possible.

Since $C_{j}^{k}$ is started at $b_{e} g^{k}$ for each $k$, it is started at least as early as any other correct $C_{i}^{k}$ in execution $e$. Furthermore, between $b e g^{k}$ and $b e g^{k+1}, C_{j}^{k}$ increases at the maximum possible rate. Hence $C_{j}^{k}$ is an upper bound on the $k$ th logical clocks of all correct processes in execution $e$. That is, for $t \in\left[e n d^{k}, e n d^{k+1}\right], C_{i}^{k}(t) \leq C_{j}^{k}(t)$, for any other correct process $i$.

We now show that $C_{j}^{k}$ is an upper bound on the $k$ th logical clock of any correct process in any execution in $E\left(t_{0}\right)$. To prove this, wc first show that, for any $k \geq 1$, ready $y^{k}$ in execution $e$ is at least as early as ready ${ }^{k}$ in any other execution $e^{\prime} \in E\left(t_{0}\right)$. The proof is by induction on $k$.

For $k=1$, ready ${ }^{1}=t_{0}$ for all executions in $E\left(t_{0}\right)$. Assume, for some $k>1$, that ready $y^{k}$ in execution $e$ is no later than ready in execution $e^{\prime}$. In execution $e$, 
$b e g^{k}=r e a d y^{k}$, the $k$ th logical clock of process $j$ is started at $b e g^{k}$, and process $j$ runs at the maximum possible rate. Therefore ready $y^{k+1}=r e a d y^{k}+(P-\alpha) /(1+$ $\rho$ ). It is easy to show that, in any execution, ready $y^{k+1} \geq$ ready $^{k}+(P-\alpha) /(1+\rho)$. Therefore ready $y^{k+1}$ in execution $e$ is at least as early as that in execution $e^{\prime}$.

In execution $e$, beg $^{k}=$ read $^{k}$ for all $k \geq 1$. By Lemma 3 , in any execution, beg ${ }^{k}$ $\geq$ ready $y^{k}$ for all $k \geq 1$. Therefore the $k$ th logical clock of process $j$ is started no later than that of any other correct process in any execution in $E\left(t_{0}\right)$. Since process $j$ also runs at the maximum possible rate, $C_{j}^{k}$ is an upper bound on the $k$ th logical clocks of all correct processes in all executions in $E\left(t_{0}\right)$.

We now estimate an upper bound for $C_{j}^{k}$. For process $j$, the interval of real time between consecutive resynchronizations is $(P-\alpha) /(1+\rho)$. In this period its logical time increases by $P$. Therefore, for all $k \geq 1$ and for $t \in\left[e n d^{k}\right.$, end $\left.{ }^{k+1}\right]$,

$$
\frac{C_{j}^{k}(t)-C_{j}^{\prime}\left(t_{0}\right)}{t-t_{0}} \leq \frac{P}{P-\alpha}(1+\rho) .
$$

Since $C_{j}^{\prime}\left(t_{0}\right)=P+\alpha$, a constant

$$
C_{j}^{k}(t) \leq \frac{P}{P-\alpha}(1+\rho) t+b,
$$

where $b$ is a constant that depends on $t_{0}$.

Lemma 10. For any execution of the algorithm of Figure 1, there exists a constant a, such that, for all correct processes $i$, all $k \geq 1$, and for $t \in\left[e n d^{k}\right.$, end $\left.{ }^{k+1}\right]$,

$$
\frac{P}{P-\alpha+\left[t_{d e l} /(1+\rho)\right]}(1+\rho)^{-1} t+a \leq C_{i}^{k}(t) .
$$

Proof. Let $F\left(t_{0}\right)$ be the set of executions of the algorithm in which end ${ }^{1}=t_{0}$. Consider an execution $e \in F\left(t_{0}\right)$ where, for all $k \geq 1$, correct process $j$ accepts the (round $k$ ) message $t_{\text {del }}$ in real time after $C_{j}^{k-1}$ reads $k P$. Also, $C_{j}^{k}$ is started at $e n d^{k}$ for all $k \geq 1$. In $e$ the physical clock of process $j$ runs at the minimum possible rate, that is, at $(1+\rho)^{-1}$ with respect to real time. Such an execution is clearly possible. It is easy to show that $C_{j}^{k}$ is a lower bound on the $k$ th logical clocks of all correct processes in execution $e$. That is, for $t \in\left[e n d^{k}\right.$, end $\left.{ }^{k+1}\right], C_{j}^{k}(t) \leq C_{i}^{k}(t)$, for any other correct process $i$.

$C_{j}^{k}$ is also a lower bound on the $k$ th logical clocks of all correct processes in any execution in $F\left(t_{0}\right)$. In execution $e$ we have end $d^{k+1}=e n d^{k}+(P-\alpha)(1+\rho)+t_{\text {del- }}$ The proof follows by Lemma 5 and an easy induction on $k$.

We now estimate a lower bound for $C_{j}^{k}$. For process $j,(P-\alpha)(1+\rho)+t_{\text {del }}$ is the interval of real time between consecutive resynchronizations. In this period, its logical time increases by $P$. Therefore, as in Lemma 9 , for all $k \geq 1$ and $t \in\left[e n d^{k}\right.$, end ${ }^{k+1}$,

$$
C_{j}^{k}(t) \geq \frac{P}{(P-\alpha)(1+\rho)+t_{\mathrm{del}}} t+a
$$

for some constant $a$ that depends on $t_{0}$.

THEOREM 1. The algorithm in Figure 1 is a synchronization algorithm. With this algorithm correct processes send a total of $O\left(n^{2}\right)$ signed messages per resynchronization. 
ProOF. By Lemma 8, the algorithm achieves agreement. To show accurcy let

$$
(1+\gamma)=\frac{P}{P-\alpha}(1+\rho) .
$$

From Lemma $9, C_{i}^{k}(t) \leq(1+\gamma) t+b$. Note that

$$
\frac{P-\alpha}{P}(1+\rho)^{-1} \leq \frac{P}{P-\alpha+\left[t_{\mathrm{del}} /(1+\rho)\right]}(1+\rho)^{-1} .
$$

Hence, from Lemma $10,(1+\gamma)^{-1} t+a \leq C_{i}^{k}(t)$, and accuracy is achieved.

In each resynchronization round, each correct process broadcasts at most one message with its own signature and one message containing the $f+1$ signed messages it needs to relay. Thus correct processes send a total of $O\left(n^{2}\right)$ messages per resynchronization.

Hence the number of messages and bits exchanged for each resynchronization is comparable to that in [7].

\subsection{ACHIEving Optimal AcCuracy}

3.3.1 A Bound on Accuracy. We first show that, for any synchronization algorithm, the accuracy of synchronized logical clocks cannot exceed that of the underlying hardware clocks.

THEOREM 2. For any synchronization algorithm, even in the absence of faults, the bound on the rate of drift of logical clocks from real time is at least as large as the bound on the rate of drift of physical clocks.

PROOF. Consider an algorithm that satisfies agreement and accuracy. For simplicity, assume that all physical clocks are set to 0 at time $t=0$, that is, $R_{i}(0)=0$ for all $i$. Then, all correct physical clocks satisfy the relation

$$
(1+\rho)^{-1} t \leq R_{i}(t) \leq(1+\rho) t .
$$

Consider an execution of the algorithm in which all processes in the system are correct and the physical clock of each process runs at the maximum possible rate. That is, for all processes $i, R_{i}^{(1)}(t)=(1+\rho) t$, where superscripts denote execution numbers. Further, assume the transmission delay for each message is exactly $d$, with $d \leq t_{\mathrm{del}} /(1+\rho)^{2}$. By accuracy, in this execution, for all correct processes $i$ and for some constant $b^{(1)}$,

$$
C_{i}^{(1)}(t) \leq(1+\gamma) t+b^{(1)} .
$$

Now consider a second execution in which all processes are still correct, but have their physical clocks running at the minimum possible rate. That is, for all processes $j, R_{i}^{(2)}(t)=(1+\rho)^{-1} t$. Let the transmission delay for each message be $d(1+\rho)^{2}$. Again, by accuracy, in this execution for all correct processes $i$ and for some constant $a^{(2)}$,

$$
(1+\gamma)^{-1} t+a^{(2)} \leq C_{i}^{(2)}(t) .
$$

Assume that, for each process $i$, the initial state is the same in both executions. That is, in both executions, a process starts executing the algorithm at the same reading of its physical clock. In the second execution, physical clocks and the speed at which messages are delivered are slowed down by the same factor, $(1+\rho)^{2}$, with respect to the first execution. Therefore, from within the system, both executions 
appear identical to every process. Hence, considering a particular process $i$, the rate at which its logical time advances with respect to its physical time must be the same in both executions. In particular, if $R_{i}^{(1)}\left(t_{1}\right)=R_{i}^{(2)}\left(t_{2}\right)$ for some $t_{1}$ and $t_{2}$, then $C_{i}^{(1)}\left(t_{1}\right)=C_{i}^{(2)}\left(t_{2}\right)$.

Since $R_{i}^{(1)}(t)=(1+\rho) t$ and $R_{i}^{(2)}(t)=(1+\rho)^{-1} t$, it follows that, if $t_{2}=(1+\rho)^{2} t_{1}$, then $R_{i}^{(1)}\left(t_{1}\right)=R_{i}^{(2)}\left(t_{2}\right)$ and therefore $C_{i}^{(1)}\left(t_{1}\right)=C_{i}^{(2)}\left(t_{2}\right)$. Therefore, from eqs. (1) and $(2),(1+\gamma) t_{1}+b^{(1)} \geq(1+\gamma)^{-1}(1+\rho)^{2} t_{1}+a^{(2)}$ for all $t_{1}$. This implies that $\gamma \geq \rho$.

3.3.2 An Algorithm for Optimal Accuracy. We now describe a modification to our algorithm to achieve optimal accuracy. In the algorithm of Figure 1, correct processes start their $k$ th clocks as soon as they accept a (round $k$ ) message. However, there is an uncertainty of $t_{\text {del }}$ in the time it takes for correct processes to accept a message. It is this uncertainty that introduces a difference in the logical time between resynchronizations. For the fastest clock, the logical time between resynchronizations is $P-\alpha$ (Lemma 9$)$, and for the slowest clock, this interval is $P-\alpha+t_{\mathrm{del}} /(1+\rho)$ (Lemma 10). Informally, we can compensate for this as follows: If a process accepts a (round $k$ ) message early, it delays the starting of the $k$ th clock by $t_{\mathrm{del}} / 2(1+\rho)$. If it accepts the message late, it advances the starting of the $k$ th clock by $t_{\text {del }} / 2(1+\rho)$. Thus, in the cases described in both Lemmas 9 and 10, the logical time between resynchronizations becomes $P-\alpha+\beta$, where $\beta=t_{\mathrm{del}} / 2(1+\rho)$. This is used to show that the drift of logical clocks is bound above by

$$
\frac{P}{P-\alpha+\beta}(1+\rho)
$$

and below by

$$
\frac{P}{P-\alpha+\beta}(1+\rho)^{-1} .
$$

By slowing down the logical clocks by a factor of $P / P-\alpha+\beta$, we obtain an algorithm where the rate of drift of logical clocks is optimal: bounded by $(1+\rho)$ above and by $(1+\rho)^{-1}$ below.

More precisely, suppose process $i$ accepts (round $k$ ) at time $t$, and let $T=C_{i}^{k-1}(t)$. If $T \leq k P+\beta$, we say the (round $k$ ) message was accepted early. Process $i$ delays the starting of $C_{i}^{k}$ by setting it to $k P+\alpha$ when $C_{i}^{k-1}$ reads $\min (T+\beta, k P+\beta)$. In this case, the start of $C_{i}^{k}$ is delayed by at most $\beta$, but never beyond the time when $C_{i}^{k-1}$ reads $k P+\beta$.

If $T>k P+\beta$, we say (round $k$ ) was accepted late. Process $i$ advances the starting of $C_{i}^{k}$, by setting it to $k P+\alpha$ when $C_{i}^{k-1}$ reads $T^{\prime}=\max (T-\beta, k P+\beta)$. Note that $C_{i}^{k}$ must be started when $C_{i}^{k-1}$ reads $T^{\prime}<T$, that is, "in the past." This is achieved by setting $C_{i}^{k}$ to $k P+\alpha+\left(T-T^{\prime}\right)$ when $C_{i}^{k-1}$ reads $T$. That is, $C_{i}^{k}$ is set to $\min \left(C_{i}^{k-1}(t)+\alpha-\beta, k P+\alpha+\beta\right)$ at time $t$. In this case, the start of $C_{i}^{k}$ is advanced by at most $\beta$, but never started before $C_{t}^{k-1}$ reads $k P+\beta$.

The definitions of ready ${ }^{k}, b_{e g}{ }^{k}$, and $e n d^{k}$ are the same as before: ready $y^{k}$ is the earliest time at which a correct process sends a (round $k$ ) message, and $b^{k} g^{k}$ and $e n d^{k}$ are the earliest and latest times at which some correct process starts its $k$ th clock (setting it to $k P+\alpha$ ).

We first show that this modified algorithm achieves agreement by showing that Lemmas 1-8 still hold. 
Proof of Lemma 1. The first correct process to start its $k$ th clock can start it $\beta$ in logical time (or $\beta(1+\rho)$ in real time) before it accepts a (round $k$ ) message. Every correct process accepts (round $k$ ) within $t_{\text {del }}$ of the first correct process accepting it and starts its $k$ th clock within a further $\beta(1+\rho)$. Therefore $e n d^{k}-$ $b e g^{k} \leq t_{\text {del }}+2 \beta(1+\rho)=2 t_{\text {del. }}$. Therefore Lemma 1 is satisfied with $d_{\min }=2 t_{\mathrm{del}}$.

Proof of Lemma 2. Same as in Section 3.

Proof of Lemma 3. Consider any correct process $i$. By definition, $C_{i}^{k-1}\left(r e a d y^{k}\right)$ $\leq k P$. Let process $i$ accept the (round $k$ ) message at real time $t$. Note that $t \geq$ ready ${ }^{k}$. If $C_{i}^{k-1}(t) \leq k P+\beta$, then process $i$ delays the starting of the $k$ th clock. If $C_{i}^{k-1}(t)>k P+\beta$, process $i$ starts its $k$ th clock no earlier than at real time $t^{\prime}$ such that $C_{i}^{k-1}\left(t^{\prime}\right)=k P+\beta$. Clearly, $t^{\prime} \geq$ read $^{k}$. Hence no correct process starts its $k$ th clock before ready ${ }^{k}$.

ProOF OF Lemma 4. Every correct process that broadcasts a (round $k$ ) message does so by real time $t_{1}=$ ready $y^{k}+(1+\rho) D_{\max }$. Therefore every correct process accepts (round $k$ ) by $t_{2}=t_{1}+t_{\text {del. }}$. For any correct process $i, C_{i}^{k-1}\left(t_{1}\right) \geq k P$, and hence $C_{i}^{k-1}\left(t_{2}\right) \geq k P+t_{\mathrm{dcl}} /(1+\rho)=k P+2 \beta$. Thus, with the modified algorithm, every correct process starts its $k$ th clock at real time $t<t_{2}$. Therefore $e n d^{k}-$ ready ${ }^{k}$ $\leq(1+\rho) D_{\max }+t_{\mathrm{del}}$.

Proof of Lemma 5. Consider any correct process $i$. Process $i$ accepts a (round $k+1)$ message by real time $t=e n d^{k}+(P-\alpha)(1-\rho)+t_{\text {del. }}$. Also, $C_{i}^{k}(t) \geq k P+t_{\mathrm{del}} /(1+\rho)$. Therefore process $i$ starts its $k+1$ st clock by real time $t$, proving the lemma.

Proofs of Lemmas 6, 7, and 8. Same as in Section 3.

Thus the modified algorithm achieves agreement. To show that the modified algorithm achieves optimal accuracy, we first evaluate the bounds on the drift of logical clocks from real time.

LEMMA 9'. For any execution of the modified algorithm, there exists a constant $d$, such that for all correct processes $i$, all $k \geq 1$, and $t \in\left[\right.$ end $^{k}$, end $\left.^{k+1}\right]$,

$$
C_{i}^{k}(t) \leq \frac{P}{P-\alpha+\beta}(1+\rho) t+d .
$$

Proof. Let $E\left(t_{0}\right)$ be the set of executions of the algorithm in which ready ${ }^{1}=t_{0}$. Consider an execution $e \in E\left(t_{0}\right)$ in which, for all $k \geq 1$, correct process $j$ broadcasts and accepts (round $k$ ) at ready $y^{k}$. In execution $e$ the physical clock of process $j$ runs at the maximum possible rate, that is, $(1+\rho)$ with respect to real time.

Process $j$ accepts (round $k$ ) at ready ${ }^{k}$ when $C_{j}^{k-1}$ reads $k P$ (i.e., early). Therefore $C_{j}^{k}$ is started at real time $t$ such that $C_{j}^{k-1}(t)=k P+\beta$, that is, when $t=r e a d y^{k}+\beta /(1+\rho)$. Note that no correct physical clock increases by more than $\beta$ between ready $y^{k}$ and $t$.

Consider another correct process $i$. By definition of ready $y^{k}, C_{i}^{k-1}\left(\right.$ ready $\left.^{h}\right) \leq k P$, and therefore $C_{i}^{k-1}(t) \leq k P+\beta$. Suppose process $i$ accepts (round $k$ ) when $C_{i}^{k-1}$ reads $T_{i}$. This must occur after ready $y^{k}$, and thercfore, at time $t, C_{i}^{k-1}(t) \leq T_{i}+\beta$. 
We consider two cases:

(1) If $T_{i} \leq k P+\beta$, then process $i$ starts $C_{i}^{k}$ at real time $t^{\prime}$ when $C_{i}^{k-1}\left(t^{\prime}\right)=$ $\min \left(T_{i}+\beta, k P+\beta\right)$. Since both $C_{i}^{k-1}(t) \leq T_{i}+\beta$ and $C_{i}^{k-1}(t) \leq k P+\beta$, then $t \leq t^{\prime}$.

(2) If $T_{i}>k P+\beta$, process $i$ starts $C_{i}^{k}$ at real time $t^{\prime}$ when $C_{i}^{k-1}\left(t^{\prime}\right)=\max \left(T_{i}-\beta\right.$, $k P+\beta)$. Therefore, $C_{i}^{k-1}\left(t^{\prime}\right) \geq k P+\beta \geq C_{i}^{k-1}(t)$ and $t^{\prime} \geq t$.

Thus, in execution $e$, for any $k \geq 1$, the $k$ th clock of process $i$ is started no earlier than that of process $j$. Between resynchronizations $C_{j}^{k}$ runs at the maximum possible rate. Therefore $C_{j}^{k}$ is an upper bound on the $k$ th logical clock of all correct processes in execution $e$. As in Lemma 9, we can also show that $C_{j}^{k}$ is an upper bound on the $k$ th clock of all correct processes in any execution in $E\left(t_{0}\right)$.

Between every two successive resynchronizations, the logical clock of process $j$ is advanced by $P$, and the time that elapses on the logical clock of $j$ is $P-\alpha+\beta$. (E.g., at the $k$ th resynchronization, the clock is set to $k P+\alpha$, the $k+1$ st resynchronization occurs when this clock reads $(k+1) P+\beta$, and the new clock is set to $(k+1) P+\alpha$.) Since the clock of process $j$ runs at $(1+\rho)$ with respect to real time, the real time that elapses between two resynchronizations is $(P-\alpha+\beta) /(1+\rho)$. Hence, for all $k \geq 1$ and $t \in\left[e n d^{k}\right.$, end $\left.d^{k+1}\right]$,

$$
C_{j}^{k}(t) \leq \frac{P}{P-\alpha+\beta}(1+\rho) t+d
$$

for some constant $d$ that depends on $t_{0}$.

LEMMA 10'. For any execution of the modified algorithm, there exists a constant $c$, such that for all correct processes $i$, all $k \geq 1$, and $t \in\left[e^{2} d^{k}\right.$, end $\left.{ }^{k+1}\right]$,

$$
\frac{P}{P-\alpha+\beta}(1+\rho)^{-1} t+c \leq C_{i}^{k}(t) \text {. }
$$

Proof. Let $F\left(t_{0}\right)$ be the set of executions of the algorithm in which end ${ }^{1}=t_{0}$. Define last ${ }^{k}$ to be the latest real time at which a correct process accepts (round $k$ ). Consider an execution $e \in F\left(t_{0}\right)$ in which the first logical clock of a correct process $j, C_{j}^{1}$ is started at $e n d^{1}$, and for all $k \geq 1$, process $j$ accepts (round $k$ ) at $l a t^{k}$, and $t_{\text {del }}$ (in real time) after its logical clock reads $k P$. The physical clock of process $j$ runs at the minimum possible rate, that is, at $(1+\rho)^{-1}$ with respect to real time. In the modified algorithm, since $C_{j}^{k-1}\left(\right.$ last $\left.^{k}\right)=k P+2 \beta$, process $j$ sets its $k$ th clock to $k P+\alpha+\beta$ at last $^{k}$.

We now show that the logical clock of process $j$ is as slow as that of any other correct process in any execution in $F\left(t_{0}\right)$. That is, we show that, for all $k \geq 1$ and $t \in\left[\right.$ last $^{k}$, last $\left.^{k+1}\right], C_{j}^{k}(t) \leq C_{i}^{k}(t)$ for any correct process $i$ in any execution in $F\left(t_{0}\right)$. The proof is by induction on $k$.

For $k=1$ note that $C_{j}^{1}$ is started at $e n d^{1}=t_{0}$, and process $j$ runs at the minimum possible rate. In any execution in $F\left(t_{0}\right)$, for any other correct process $i, C_{i}^{1}$ is started no later than at end $d^{1}$. Therefore, for $t \geq e n d^{1}$, and specifically for $t \in\left[\right.$ last $^{1}$, last $\left.{ }^{2}\right]$, we see that $C_{j}^{1}(t) \leq C_{i}^{1}(t)$. For the inductive step, assume that, for some $k>1$ and $t \in\left[\right.$ last $^{k-1}$, last $\left.{ }^{k}\right]$, we have $C_{j}^{k-1}(t) \leq C_{i}^{k-1}(t)$ for any correct process $i$. Define $s_{i}$ to be the real time such that $C_{i}^{k-1}\left(s_{i}\right)=k P+\beta$, for any process $i$. Let $t_{i}$ and $T_{i}$ be the real and the corresponding logical time at which a process $i$ accepts (round $k$ ).

Consider any correct process $i$ in any execution in $F\left(t_{0}\right)$. From the induction hypothesis, it follows that $s_{i} \leq s_{j}$ for all correct $i$. Since $s_{j}=$ last ${ }^{k}-\beta(1+\rho)$, last $^{k}-s_{i} \geq \beta(1+\rho)$. By assumption, $t_{j}=$ last $^{k}$ and $T_{j}=k P+2 \beta$. We consider two 
cases:

(1) If $T_{i} \leq k P+\beta$ (i.e., $t_{i} \leq s_{i} \leq s_{j}$ ), then $C_{i}^{k}$ is set to $k P+\alpha$ no later than $s_{i}$. Since last $^{k}-s_{i} \geq \beta(1+\rho), C_{i}^{k}$ increases by at least $\beta$ between $s_{i}$ and last ${ }^{k}$. Therefore $C_{i}^{k}\left(\right.$ last $\left.^{k}\right) \geq k P+\alpha+\beta=C_{j}^{k}\left(\right.$ last $\left.^{k}\right)$.

(2) If $T_{i}>k P+\beta$, then process $i$ sets its $k$ th clock to $C_{i}^{k}\left(t_{i}\right)=\min \left(C_{i}^{k-1}\left(t_{i}\right)+\alpha-\right.$ $\beta, k P+\alpha+\beta)$. Since $C_{i}^{k}$ and $C_{i}^{k-1}$ increase by the same amount between $t_{i}$ and last $^{k}, C_{i}^{k}\left(\right.$ last $\left.^{k}\right)=\min \left(C_{i}^{k-1}\left(t_{i}\right)+\alpha-\beta, k P+\alpha+\beta\right)+C_{i}^{k-1}\left(\right.$ last $\left.^{k}\right)-$ $C_{i}^{k-1}\left(t_{i}\right)$. Since $C_{i}^{k-1}\left(\right.$ last $\left.^{k}\right) \geq k P+2 \beta, C_{i}^{k}\left(\right.$ last $\left.^{k}\right) \geq k P+\alpha+\beta=C_{j}^{k}\left(\right.$ last $\left.^{k}\right)$.

Thus $C_{j}^{k}\left(\right.$ last $\left.^{k}\right) \leq C_{i}^{k}\left(\right.$ last $\left.^{k}\right)$. The physical clock of process $j$ runs at the minimum possible rate. Therefore, for $t \in\left[\right.$ last $^{k}$, last $\left.{ }^{k+1}\right], C_{j}^{k}(t) \leq C_{i}^{k}(t)$ for any correct process $i$ in any execution in $F\left(t_{0}\right)$.

The logical clock of process $j$ is incremented by $P$ over successive resynchronizations. The real time that elapses between successive resynchronizations of process $j$ is $(P-\alpha+\beta)(1+\rho)$. Thus, for any execution of the modified algorithm, there exists a constant $c$ (that depends on $t_{0}$ ), such that for all correct processes $i$, all $k \geq 1$, and $t \in\left[\right.$ last $^{k}$, last $\left.^{k+1}\right]$,

$$
\frac{P}{P-\alpha+\beta}(1+\rho)^{-1} t+c \leq C_{j}^{k}(t) .
$$

Since for $t \in\left[\right.$ end $^{k}$, last $\left.{ }^{k}\right] C_{j}^{k}(t) \geq C_{j}^{k-1}(t)$, the above inequality also holds for $t \in\left[\right.$ end $^{k}$, end $\left.{ }^{k+1}\right]$.

By Lemmas $9^{\prime}$ and $10^{\prime}$, in any execution of the algorithm, for $k \geq 1$ and $t \in\left[e n d^{k}\right.$, end $\left.{ }^{k+1}\right]$, the logical clock of any correct process $i$ is within the envelope

$$
\mu(1+\rho)^{-1} t+c \leq C_{i}^{k}(t) \leq \mu(1+\rho) t+d,
$$

where $\mu=P /(P-\alpha+\beta)$, and $c$ and $d$ are constants depending on the initial conditions of this execution. Therefore

$$
(1+\rho)^{-1} t+\frac{c}{\mu} \leq \frac{C_{i}^{k}(t)}{\mu} \leq(1+\rho) t+\frac{d}{\mu} .
$$

Hence, if correct processes slow down their logical clocks by this factor of $\mu$, that is, process $i$ uses $L_{i}^{k}(t)=C_{i}^{k}(t) / \mu$ as its logical time, optimal accuracy is achieved. Also, since $\mu>1$, agreement is still guaranteed. Process $i$ continues to use $C_{i}^{k}$ for the synchronization algorithm.

THEOREM 3. With the modification described above, the algorithm of Figure 1 achieves optimal accuracy.

ProOF. Follows from the above discussion.

3.4 Bounds on Faults Tolerated. Consider a system with a weak type of failure: A process is faulty only by violating assumption A1 (i.e., its physical clock may run slower or faster than the specified bound). We now show that even with this weak type of failure, optimal accuracy cannot be achieved unless fewer than half the processes are faulty.

THEOREM 4. Any synchronization algorithm that achieves optimal accuracy must have a majority of correct clocks.

Proof. Assume that there exists a synchronization algorithm that achieves optimal accuracy for systems with $n \leq 2 f$. We show that this is impossible by first 
considering a system with two processors $p_{1}$ and $p_{2}$, one of which can be faulty (i.e., $n=2$ and $f=1$ ).

Since the algorithm achieves optimal accuracy, in any execution of the algorithm, the logical clock of correct process $i$ satisfies the following relation for all $t \geq e n d^{1}$ :

$$
(1+\rho)^{-1} t+a \leq C_{i}(t) \leq(1+\rho) t+b,
$$

where $a$ and $b$ are constants. Also, since the algorithm achieves agreement, there exists a constant $D_{\max }$ such that, if $p_{1}$ and $p_{2}$ are correct, then $\left|C_{1}(t)-C_{2}(t)\right| \leq$ $D_{\max }$ for all $t \geq e n d^{1}$.

We now consider three possible executions of the algorithm. In what follows, superscripts correspond to execution numbers. For simplicity, we assume that all physical clocks start at 0 at real time 0 . Assume that the initial state of a given process is the same in all executions. That is, a given process starts executing the algorithm at the same reading of its physical clock.

Execution $e_{1}$. Both processes are correct. The physical clock of $p_{1}$ runs at the maximum rate possible and that of $p_{2}$ at the minimum rate possible. That is, $R_{1}^{(1)}(t)=(1+\rho) t$ and $R_{2}^{(1)}(t)=(1+\rho)^{-1} t$. The transmission time for each message is exactly $d$, where $d \leq t_{\text {del }} /(1+\rho)^{2}$.

Execution $e_{2}$. Process $p_{1}$ is correct, and the rate of its physical clock is given by $R_{1}^{(2)}(t)=(1+\rho)^{-1} t$. The clock of $p_{2}$ is faulty and runs at $R_{2}^{(2)}(t)=(1+\rho)^{-3} t$, but $p_{2}$ is otherwise correct and follows the algorithm. The transmission time of each message is $d(1+\rho)^{2}$.

Execution $e_{3}$. Process $p_{2}$ is correct, and its physical clock is given by $R_{2}^{(3)}(t)=$ $(1+\rho) t$. The clock of $p_{1}$ is faulty and runs at $R_{1}^{(3)}(t)=(1+\rho)^{3} t$, but $p_{1}$ is otherwise correct. All messages now take $d /(1+\rho)^{2}$ to be delivered.

We see that all three executions are possible. Since optimal accuracy is achieved and $p_{1}$ is correct in $e_{1}$, its logical clock satisfies the relation $C_{1}^{(1)}(t) \leq(1+\rho) t+b^{(1)}$. Since $R_{1}^{(1)}(t)=(1+\rho) t$, we see that $C_{1}^{(1)}(t) \leq R_{1}^{(1)}(t)+b^{(1)}$. Similarly, in execution $e_{2}$, we see that $R_{1}^{(2)}(t)+a^{(2)} \leq C_{1}^{(2)}(t)$. But the two executions look identical to $p_{1}$, and hence the relation between its logical and physical clocks must be the same in both executions. Therefore, to satisfy the two relations above, we see that for $k=1,2$,

$$
R_{1}^{(k)}(t)+a^{(2)} \leq C_{1}^{(k)}(t) \leq R_{1}^{(k)}(t)+b^{(1)} .
$$

Therefore, in execution $e_{1}$, there exists a time $\tau$ such that for all $t \geq \tau$

$$
(1+\rho) t+a^{(2)} \leq C_{1}^{(1)}(t) \leq(1+\rho) t+b^{(1)} \text {. }
$$

Similarly, by considering executions $e_{1}$ and $e_{3}$, in both of which $p_{2}$ is correct, we see that there exists a time $\tau^{\prime}$ such that for all $t \geq \tau^{\prime}$

$$
(1+\rho)^{-1} t+a^{(1)} \leq C_{2}^{(1)}(t) \leq(1+\rho)^{-1} t+b^{(3)}
$$

From these two relations, it follows that in execution $e_{1}$, for any given $D_{\max }$, there is some time $t^{\prime}$ such that, for all $t \geq t^{\prime}$, the deviation between the two correct logical clocks is greater than $D_{\max }$, which violates the agreement condition.

This can be generalized to any system of $n \geq 2$ processes, where $n \leq 2 f$. Partition the processes into two sets $P_{1}$ and $P_{2}$, with not more than $f$ processes in either set. By constructing executions similar to those above, we can prove that no synchronization algorithm can achieve optimal accuracy if $n \leq 2 f$. 
The authenticated algorithm of Figure 1 requires $n>2 f$ processes. By Theorem 3, this algorithm can be modified to achieve optimal accuracy. From Theorem 4, it follows that the modified algorithm is also optimal in the number of faults tolerated.

\section{Synchronization without Authentication}

4.1 Simulating AuthentiCated BroadCasts. The proof of correctness and the analysis of the authenticated algorithm rely on the following properties of the message system:

P1. Correctness. If at least $f+1$ correct processes broadcast (round $k$ ) messages by time $t$, then every correct process accepts the message by time $t+t_{\text {del. }}$.

P2. Unforgeability. If no correct process broadcasts a (round $k$ ) message by time $t$, then no correct process accepts the message by $t$ or earlier.

P3. Relay. If a correct process accepts the message (round $k$ ) at time $t$, then every correct process does so by time $t+t_{\mathrm{del}}$.

As seen earlier, implementing authentication using digital signatures provides these three properties. However, the correctness of the algorithm does not depend on this particular implementation, and any other implementation providing these properties can be used instead. A broadcast primitive to simulate authentication is described in [14]. By replacing authenticated broadcasts in the algorithm of Figure 1 with this primitive, we get a logically equivalent nonauthenticated algorithm having the properties of the authenticated algorithm. However, the number of messages sent by correct processes is $O\left(n^{3}\right)$ per resynchronization.

We now modify this broadcast primitive to achieve the three properties described above at a cost of only $O\left(n^{2}\right)$ messages per resynchronization. The primitive is presented in Figure 2 and requires $n \geq 3 f+1$. With this primitive, each broadcast now requires two phases of communication. Therefore, $t_{\mathrm{del}}$, the upper bound on the time required for a message to be prepared by a process, sent to all processes, and processed by the correct processes accepting it, must be reevaluated. Let $\tau$ be the maximum transmission delay between any two processes. Then, $t_{\text {del }} \geq 2 \tau$.

THEOREM 5. The broadcast primitive achieves properties of correctness, unforgeability, and relay. The number of messages sent by correct processes is $O\left(n^{2}\right)$ per resynchronization.

\section{ProOF}

Correctness. Since at least $f+1$ correct processes broadcast (round $k$ ) by time $t$, every correct process receives at least $f+1$ (init, round $k$ ) messages by time $t+\tau$ and sends (echo, round $k$ ). Hence, by time $t+2 \tau$, every correct process receives at least $2 f+1$ (echo, round $k$ ) messages. That is, every correct process accepts (round $k$ ) by time $t+t_{\mathrm{del}}$.

Unforgeability. Since no correct process sends an (init, round $k$ ) message by time $t$, a correct process could have received (init, round $k$ ) messages from at most $f$ processes and (echo, round $k$ ) messages from at most $f$ processes. Thus no correct process sends an (echo, round $k$ ) message by time $t$. Hence no correct process accepts (round $k$ ) by time $t$.

Relay. Since a correct process accepts (round $k$ ) at time $t$, it must have received at least $2 f+1$ (echo, round $k$ ) messages. Every correct process receives at least 
To broadcast a (round $k$ ) message, a correct process sends (init, round $k$ ) to all.

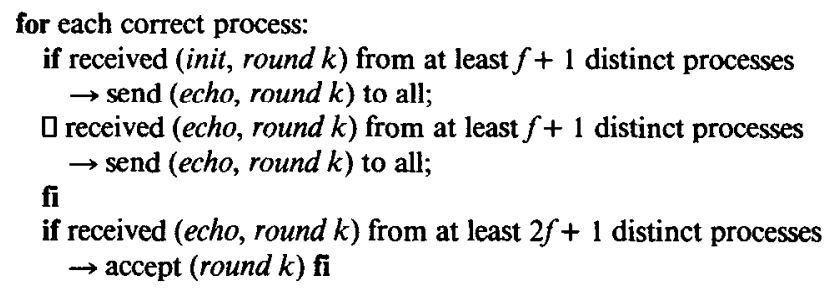

FIG. 2. A broadcast primitive to achieve properties P1, P2, and P3.

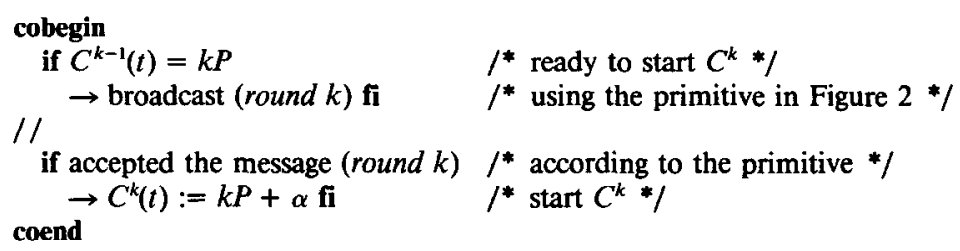

Fig. 3. A nonauthenticated algorithm for clock synchronization for process $p$ for round $k$.

$f+1$ of these within another $\tau$ and sends an (echo, round $k$ ) if it has not already done so. Hence, by $t+2 \tau$ (i.e., by $t+t_{\text {del }}$ ), every process accepts a (round $k$ ) message.

Since each correct process sends at most two messages for each resynchronization round (an init and an echo), the total number of messages sent by correct processes is at most $2 n^{2}$ per round.

4.2 A Nonauthenticated Algorithm for Clock Synchronization. Replacing signed communication with our broadcast primitive extends the synchronization algorithm of Figure 1 to one for systems without authentication. The relay property of the primitive implies that we need not explicitly relay messages since the primitive does this automatically. Since the primitive requires $n>3 f$, the nonauthenticated algorithm also has this limit on the number of faulty processes. It has been shown that, if authentication is not available, then synchronization is impossible unless $n>3 f[3,5]$.

As in Section 2, we assume that clocks are initially synchronized such that, at ready ${ }^{1}$, all correct processes are using $C^{0}$ and these clocks are at most $D_{\max }$ apart. The nonauthenticated algorithm is described in Figure 3.

THEOREM 6. The nonauthenticated algorithm in Figure 3 achieves agreement and accuracy. Correct processes send $O\left(n^{2}\right)$ messages per resynchronization.

Proof. By properties P1-P3 of the primitive of Figure 2, it is easy to see that the proofs of Lemmas 1-10 and Theorem 1 hold. Also, by Theorem 5, correct processes send $O\left(n^{2}\right)$ messages for each resynchronization round.

Thus the number of messages sent by correct processes for each resynchronization is similar to that in [10].

In Section 3.3 we showed how the authenticated algorithm could be modified to achieve optimal accuracy. Translating this modified algorithm with our broadcast primitive results in a nonauthenticated algorithm that achieves optimal accuracy. 


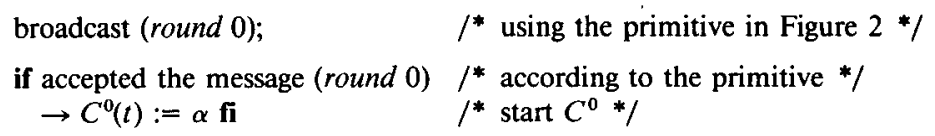

Fig. 4. A nonauthenticated algorithm for achieving initial synchronization.

send ( joining) to all processes; accept a (round $i$ ) message for some $i$;

if accepted the message (round $i+1)$ $\rightarrow C^{i+1}(t):=(i+1) P+\alpha \mathrm{fi}$

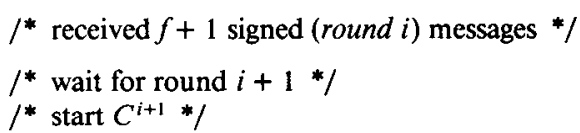

FIG. 5. A nonauthenticated algorithm used by a process to join the system.

\section{Initialization and Integration}

The algorithms presented in the previous sections can be used, with simple modifications, to achieve initial synchronization and to integrate new processes into the network.

Here we show how processes start their 0th clocks close to each other. A process decides, independently, that it is time to start clock $C^{0}$ and broadcasts a (round 0 ) message. On accepting a (round 0 ) message at real time $t$, it starts $C^{0}$ by setting $C^{0}(t)=\alpha$. The number of processes required and the rules for accepting messages are as described in Sections 2 and 4, for the authenticated and nonauthenticated systems, respectively. Since the authenticated and nonauthenticated algorithms are equivalent, we illustrate only the nonauthenticated version here (Figure 4).

It is easy to see that all processes start $C^{0}$ within $t_{\text {del }}$ of each other. Also no correct process starts $C^{0}$ until at least one correct process is ready to do so. Once they have started $C^{0}$, processes run the resynchronization algorithm. At ready ${ }^{1}$, which by definition is the time when the first correct process sends a (round 1) message, every correct logical clock reads $P$ or less. That is, every correct process is using $C^{0}$. By proofs similar to those in Lemmas 2 and 6 , it can be seen that at ready ${ }^{1}$ correct clocks are no more than $D_{\max }$ apart. Thus this algorithm justifies assumptions $\mathrm{S} 1$ and $\mathrm{S} 2$ for $k=1$ in the proof of Lemma 8.

We now describe how a process joins a system of synchronized clocks. This could be used by new processes to enter the system, or by processes that have become unsynchronized (possibly due to failures) to reestablish synchronization with the rest of the system. The algorithms are based on the idea in [10], modified to the context of our algorithms.

When a process $p$ wishes to join the system, it sends a message (joining) to the processes already in the system. It then receives messages from these processes and determines the number $i$ of the round being executed. Since $p$ could have started this algorithm in the middle of a resynchronization period, it waits for resynchronization period $i+1$ and starts its logical clock $C^{i+1}$ when it accepts a (round $i+1)$ message. It is easy to prove that its clock is now synchronized with respect to the clocks already in the system. Process $p$ now begins to run the resynchronization algorithm described earlier. We present only the nonauthenticated version in Figure 5. This algorithm can also be modified as described in Section 3.3 to ensure that optimal accuracy is achieved.

This integration scheme prevents a (possibly faulty) process joining the system from affecting the correct processes already in the system. Hence we prefer this "passive" scheme to that presented in [7]. However, with our method, a joining process might have to wait longer than in [7] before its clock is synchronized. 
To broadcast a (round $k$ ) message, a correct process sends (init, round $k$ ) to all.

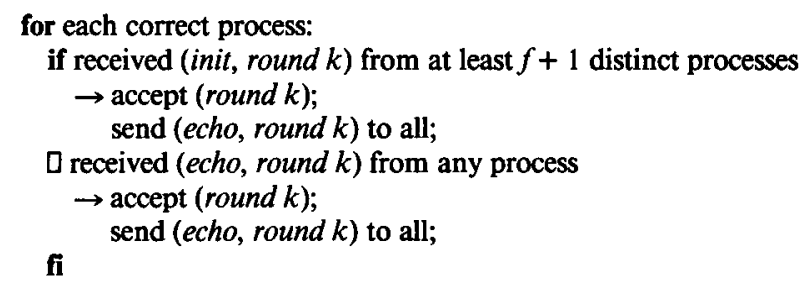

FIG. 6. A broadcast primitive to achieve properties P1, P2, and P3 for a system with sr-omission failures.

\section{Restricted Models of Failure}

In the preceding sections we have assumed that faulty processes can exhibit arbitrary behavior. Fault-tolerant algorithms have also been studied under simpler, more restrictive models of failure. It is likely that, in certain applications, faults are not as arbitrary as we have assumed so far. In such cases, developing algorithms for the simpler model of failure could result in easier and less expensive solutions.

The most benign type of failure is that of crash faults, where processes fail by just stopping $[6,8]$. Less restrictive models are omission, where faulty processes occasionally fail to send messages [6], and sr-omission, where faulty processes fail to send or receive messages [13]. In this section we show how the algorithms developed so far can be adapted to these models.

The algorithm of Figure 1 was shown to overcome arbitrary failures. The proof relied on an authenticated message system providing properties P1-P3. Consider systems with sr-omission failures, where a process is faulty either because it occasionally fails to send or receive messages, or because its physical clock does not satisfy assumption Al (i.e., they violate the specified bounds on the rate of drift from real time). For such systems we can achieve properties P1-P3 without authentication, using the broadcast primitive of Figure 6. With this broadcast primitive, the algorithm of Figure 3 is a synchronization algorithm for systems with sr-omission faults. Since crash faults and omission faults are a proper subset of sr-omission faults, the algorithm of Figure 3 can also tolerate these faults. As explained in Section 3.3, this algorithm is easily modified to achieve optimal accuracy. The primitive in Figure 6 requires $n>2 f$ processes. A broadcast by a correct process is accepted by all the correct processes within $\tau$, and hence $t_{\mathrm{del}}=\tau$. In contrast, the primitive of Figure 2 requires $n>3 f$ processes and $t_{\text {del }}=2 \tau$, but it overcomes arbitrary failures.

As seen in Section 3.4, the lower bound proofs of Theorem 2 do not assume any process or clock failures, and Theorem 4 holds even if only clocks fail. Thus our synchronization algorithm is optimal in the number of faults that can be tolerated for all the models of failure we consider.

Initial synchronization and integration of new clocks are achieved as in previous sections.

\section{Maintaining Continuous Clocks}

To simplify the presentation and analysis, we adopted the standard convention that a process starts a new logical clock after each resynchronization [7]. When a new clock is started, it is set to a value greater than that shown by the previous clock, thus ensuring that clocks are never set back. For some applications this 
scheme has two shortcomings. Since a process starts several clocks, there is ambiguity as to which clock a process should use when an external application requests the time. Moreover, setting the clock forward at each resynchronization introduces a discontinuity in the logical time (when a process switches to a new logical clock). As Lamport and Melliar-Smith noted in [9], an algorithm for discontinuously resynchronizing clocks can be easily transformed into one where logical clocks are continuous. This can be achieved by spreading out each resynchronization adjustment over the next resynchronization period. In this section we provide details on how to modify our algorithm so that each process can maintain a single continuous logical clock. This also removes ambiguity as to which clock is in use at any given time.

Each process $i$ runs the algorithm described in Section 3, maintaining its logical clocks $C_{i}^{k}$. Let $t_{i}^{k}$ be the real time of the $k$ th resynchronization of process $i$, that is, the time at which process $i$ starts the new clock $C_{i}^{k}$. The logical time of process $i$ is given by

$$
C_{i}^{k}(t)=C_{i}^{k}\left(t_{i}^{k}\right)+R_{i}(t)-R_{t}\left(t_{i}^{k}\right) \quad \text { for } \quad t_{i}^{k} \leq t \leq t_{i}^{k+1},
$$

where $R_{i}(t)$ is the value of the physical clock of process $i$ at time $t$. Let $\Delta_{i}^{k}$ be the forward adjustment that process $i$ makes to its logical clock at the $k$ th resynchronization, namely, $\Delta_{i}^{k}=C_{i}^{k}\left(t_{i}^{k}\right)-C_{i}^{k-1}\left(t_{i}^{k}\right)$. We have the following:

$$
C_{i}^{k}(t)=C_{i}^{k-1}\left(t_{i}^{k}\right)+\Delta_{i}^{k}+R_{i}(t)-R_{i}\left(t_{i}^{k}\right) \quad \text { for } \quad t_{i}^{k} \leq t \leq t_{i}^{k+1} .
$$

Using the logical clocks, $C_{i}^{k}$, we can define a single continuous clock $C_{i}$ for process $i$ as follows:

$$
\begin{array}{ll}
C_{i}(t)=C_{i}^{0}(t) & \text { for } t \leq t_{i}^{1}, \\
C_{i}(t)=C_{i}\left(t_{i}^{k}\right)+x_{i}^{k}(t) \Delta_{i}^{k}+R_{i}(t)-R_{i}\left(t_{i}^{k}\right) & \text { for } t_{i}^{k} \leq t \leq t_{i}^{k+1},
\end{array}
$$

where $x_{i}^{k}(t)=\min \left(1, R_{i}(t)-R_{i}\left(t_{i}^{k}\right) / P-\alpha-D_{\max }\right)$.

We now show that, at the $k$ th resynchronization, the continuous clock $C_{i}$ matches the logical clock $C_{i}^{k-1}$. That is, for all $k \geq 1$

$$
C_{i}\left(t_{i}^{k}\right)=C_{i}^{k-1}\left(t_{i}^{k}\right)
$$

The proof is by induction on $k$. For $k=1$ this is obvious from the definition of $C_{i}(t)$. Suppose ( $\left.{ }^{* *}\right)$ holds. From the definition of $C_{i}(t)$, we have the following:

$$
C_{i}\left(t_{i}^{k+1}\right)=C_{i}\left(t_{i}^{k}\right)+x_{i}^{k}\left(t_{i}^{k+1}\right) \Delta_{i}^{k}+R_{i}\left(t_{i}^{k+1}\right)-R_{i}\left(t_{i}^{k}\right) .
$$

By induction hypothesis, $C_{i}\left(t_{i}^{k}\right)=C_{i}^{k-1}\left(t_{i}^{k}\right)$. It is also easy to see that $x_{i}^{k}\left(t_{i}^{k+1}\right)=1$, since $R_{i}\left(t_{i}^{k+1}\right)-R_{i}\left(t_{i}^{k}\right) \geq P-\alpha-D_{\max }$. Hence from $\left({ }^{*}\right)$ we have $C_{i}\left(t_{i}^{k+1}\right)=$ $C_{i}^{k}\left(t_{i}^{k+1}\right)$, and the proof is complete.

We can easily show that the continuous clock $C_{i}(t)$ satisfies both the agreement and optimal accuracy properties. In fact, for all $t \geq 0,\left|C_{i}(t)-C_{j}(t)\right| \leq D_{\max }^{\prime} \leq$ $D_{\max }+\alpha$. Furthermore, the optimal accuracy of $C_{i}$ follows immediately from the fact that $\left|C_{i}(t)-C_{i}^{k}(t)\right|$ is bounded by $\Delta_{i}^{k} \leq D_{\max }+\alpha$, for all $k$ and all $t_{i}^{k} \leq t \leq t_{i}^{k+1}$.

\section{Discussion}

The requirements of synchronization can also be stated as follows [3, 7]: There exist constants $d_{\min }, P, D_{\max }$, and $\mathrm{ADJ}$, such that clocks are resynchronized at logical times that are multiples of $P$, and for all correct clocks $i$ and $j$ and 
all $k \geq 1$ :

C1. $\forall t \in\left[\right.$ end $^{k}$, end $\left.^{k+1}\right]$

$$
\left|C_{i}^{k}(t)-C_{j}^{k}(t)\right| \leq D_{\max }
$$

C2. If $C_{i}^{k}$ is started at time $t$, then

$$
0 \leq C_{i}^{k}(t)-C_{i}^{k-1}(t) \leq \mathrm{ADJ} .
$$

C3. $0 \leq e n d^{k}-b e g^{k} \leq d_{\text {min. }}$.

These conditions assert that the maximum deviation between correct clocks is bounded, the amount by which clocks are readjusted is bounded, and the size of a resynchronization period is small. Our algorithms satisfy these conditions. Lemmas 1 and 6 show that conditions $C 1$ and C3 are satisfied. From Lemma 4, we see that clocks are never set back. It is easy to show that the maximum adjustment made is $\alpha+D_{\max }$. Hence, by setting ADJ $=\alpha+D_{\max }$, condition C2 is also met.

A feature of our algorithm is that $d_{\min }, P$, and ADJ depend only on the system parameters $\rho$ and $t_{\mathrm{del}}$, and on the constraint $D_{\max }$. In the authenticated algorithm in [7], the adjustment ADJ is proportional to the number of faulty processes. Our solution does not use averaging, and for the nonauthenticated case, given $D_{\max }$, the maximum permitted deviation between correct clocks, our algorithm needs about half as many resynchronizations as in the best previous result [10]. The minimum value of $D_{\max }$ that our algorithm can achieve depends only on $\rho$ and $t_{\mathrm{del}}$. In [9], the minimum $D_{\max }$ possible is proportional to the number of processes in the system.

In the preceding sections, we have assumed a completely connected network. This assumption can be relaxed using well-known techniques. For an authenticated system, node connectivity of $f+1$ is sufficient. This ensures that there is at least one fault-free path between every pair of correct processes. As in [7], by defining $t_{\text {del }}$ to be the maximum time to transmit a message between correct processes along at least one fault-free path in the network, the results of Section 2 hold.

Similarly, a nonauthenticated system with node connectivity of $2 f+1$ provides at least $f+1$ distinct fault-free paths between each pair of correct processes. Define $t_{\text {del }}$ to be twice the maximum time taken for a message to be relayed along $f+1$ fault-free paths. Again, the results proved earlier for the nonauthenticated system hold.

\section{Conclusion}

In this paper we have presented a unified solution to the problems of synchronizing clocks, initializing these clocks, and integrating new clocks, for systems with different types of failures: crash, omission, and arbitrary failures with and without message authentication. This solution was derived with the help of the methodology described in [14].

This is the first known solution that achieves optimal accuracy, that is, the accuracy of synchronized clocks (with respect to real time) is as good as that specified for the underlying hardware clocks. The algorithms presented are also optimal with respect to the number of faulty processes that can be tolerated to achieve this accuracy.

In another paper [1], we describe some initial experimental results from an implementation of this algorithm on a collection of workstations connected by a local-area broadcast network. The version that we implemented overcomes arbitrary process and clocks faults. Our experience shows that these Byzantine faults 
are not necessarily expensive to overcome: The algorithm is simple, efficient, and easy to implement. Our initial results indicate that it can form the basis of an accurate, reliable, and practical distributed time service.

ACKNOWLEDGMENTS. We are grateful to Abha Moitra, Jennifer Lundelius, and the referees for their helpful comments and suggestions.

\section{REFERENCES}

1. BECK, M., SRIKANTH, T. K., AND TOUEG, S. Implementation issues in clock synchronization. In Proceedings of the Asilomar Workshop on Fault Tolerant Distributed Computing. Springer-Verlag, New York. To be published.

2. Diffie, W., and Hellman, M. New directions in cryptography. IEEE Trans. Inf. Theory IT-22 (1976), 644-654.

3. Dolev, D., Halpern, J. Y., AND Strong, R. On the possibility and impossibility of achieving clock synchronization. In Proceedings of the 16th Annual ACM STOC (Washington D.C., Apr.). ACM, New York, 1984, pp. 504-511. (Also to appear in J. Comput. Syst. Sci.)

4. DRummond, R. Impact of communication networks on fault-tolerant distributed computing. Ph.D. dissertation, Dept. of Computer Science, Cornell Univ., Ithaca, N.Y., 1986.

5. FisChER, M., LYNCH, N., AND MERRITT, M. Easy impossibility proofs for distributed consensus problems. In Proceedings of the 4th Symposium on the Principles of Distributed Computing (Minaki, Canada, Aug.). ACM, New York, 1985, pp. 59-70.

6. Hadzilacos, V. Byzantine agreement under restricted types of failures (not telling the truth is different from telling lies). Tech. Rep. 19-83, Aiken Computation Laboratory, Harvard Univ., Cambridge, Mass., June 1983.

7. Halpern, J. Y., Simons, B., Strong, R., And Dolev, D. Fault-tolerant clock synchronization. In Proceedings of the 3rd Annual ACM Symposium on Principles of Distributed Computing (Vancouver, Canada, Aug.). ACM, New York, 1984, pp. 89-102.

8. LAMPORT, L., AND FISCHER, M. Byzantine generals and transaction commit protocols. Opus 62, SRI International, Menlo Park, Calif., Apr. 1982.

9. LAMPORT, L., AND MELliaR-Smith, P. M. Synchronizing clocks in the presence of faults. J. $A C M$ 32, 1 (Jan. 1985), 52-78.

10. LUNDELIUS, J., AND LYNCH, N. A new fault-tolerant algorithm for clock synchronization. In Proceedings of the 3rd Annual ACM Symposium on Principles of Distributed Computing (Vancouver, Canada, Aug.). ACM, New York, 1984, pp. 75-88.

11. Mahaney, S. R., AND SCHNEIDER, F. B. Inexact agreement: Accuracy, precision and graceful degradation. In Proceedings of the 4th Symposium on the Principles of Distributed Computing (Minaki, Canada, Aug.). ACM, New York, 1985, pp. 237-249.

12. Marzullo, K. Maintaining the time in a distributed system. An example of a loosely-coupled distributed service. Ph.D. dissertation, Dept. of Electrical Engineering, Stanford Univ., Stanford, Calif., 1984.

13. Perry, K. J., AND Toueg, S. Distributed agreement in the presence of processor and communication faults. IEEE Trans. Softw. Eng. SE-12, 3 (Mar. 1986), 477-482.

14. SRIKanth, T. K., and Toueg, S. Simulating authenticated broadcasts to derive simple faulttolerant algorithms. Tech. Rep. 84-623, Dept. of Computer Science, Cornell Univ., Ithaca, N.Y., July 1984. (Also to appear in Distributed Computing, Springer-Verlag, New York.) 